\title{
ASPECTS DE LA TECHNIQUE DE TRADUCTION DANS UN TEXTE BILINGUE DE PANAÏT ISTRATI
}

\author{
ASPECTS OF THE TRANSLATION TECHNIQUE \\ IN A BILINGUAL TEXT OF PANAIT ISTRATI
}

\author{
Liliana AGACHE 1 \\ https://doi.org/10.52744/9786062613242.07
}

\begin{abstract}
Résumé: C'est une véritable gageure pour un.e auteur.e de traduire ses propres ouvrages, que ce soit de sa langue maternelle à sa langue d'adoption, ou de sa langue d'adoption à sa langue maternelle. Il s'agira ici d'examiner les procédés utilisés par Panaït Istrati dans sa réécriture du roman Codine (1926), rédigé en français, en un roman roumain, Codin (1935). Nous serons attentifs, en particulier, à ses stratégies d'adaptation, à l'introduction de marques de réserve subjective, ainsi qu'aux modifications sémantiques et structurelles dues au conditionnement socio-culturel propre à chaque langue.
\end{abstract}

Mots-clés: Istrati; Traduction; Adaptation; Modulation; Charge sémantique

\begin{abstract}
It is a challenge for an author to translate his or her own works, be it from mother tongue to adopted language, or from adopted language to mother tongue. The aim here is to examine the devices used by Panait Istrati in his rewriting of the novel Codine (1926), written in French, into a Romanian novel, Codin (1935). We will be attentive, in particular, to his strategies of adaptation, to the introduction of marks of subjective reservation, as well as to the semantic and structural modifications due to the socio-cultural conditioning peculiar to each language.
\end{abstract}

Keywords: Istrati; Translation; Adaptation; Modulation; Semantic Load

\section{Introduction}

Évoquer les techniques de traduction, c'est envisager des procédés variés de transposition d'un contenu linguistique et culturel spécifiques dans une autre langue et une autre culture spécifiques. Quels sont ces procédés lorsqu'il s'agit d'un auteur qui se traduit lui-même? Telle est la question à laquelle nous essayerons de répondre dans cette étude en examinant le cas de Panaït Istrati.

Panaït Istrati, écrivain roumain d'expression française, est en effet aussi l'auteur de la transposition de certaines de ses œuvres dans sa langue maternelle. Constamment insatisfait de ses traducteurs roumains, il se met

${ }^{1}$ Maître de conférence, Université Christian Dimitrie Cantemir, liliana.agache@yahoo.fr 
lui-même à la tâche. Naissent ainsi toute une série d' " équivalents » roumains des romans français : Mos Anghel en 1925 (traduction de L'Oncle Anghel, 1924), Chira Chiralina en 1934 (traduction de Kyra Kyralina, 1924), Casa Thuringer en 1933 (traduction de La maison Thüringer, 1933) et, parmi eux, Codin en 1935 (traduction de Codine, 1926), oeuvre qui fera ici l'objet de notre analyse.

Dans une étude antérieure ${ }^{2}$, j'ai montré que l'art de la modulation était le procédé prédominant dans la traduction de l'Oncle Anghel (qui deviendra en roumain Moş Anghel). Dans Codin, en revanche et comme nous allons le montrer, c'est l'adaptation qui représente la stratégie la plus fréquemment utilisée par Istrati dans son processus de décodage sémantique du texte français (Cristea 1982, 231-233). L'adaptation met en relation deux structures linguistiques conditionnées d'un point de vue socio-culturel et représente la traduction d'une unité renvoyant à des éléments péri-linguistiques ${ }^{3}$ propres à une communauté historique bien constituée socialement.

\section{Les solutions adoptées par Panaït Istrati}

Nous présentons ci-dessous les solutions proposées par Panaït Istrati lors de la transposition du français en roumain, de façon à valoriser les unités qui portent l'empreinte sociolinguistique de chacune de ces deux langues. La caractéristique la plus frappante, lorsque nous comparons les deux versions, est la différence dans la manifestation des marqueurs de réserve subjective, car ceux-ci résultent souvent du choix d'une unité lexicale présente dans une langue et n'existant pas sous cette forme dans l'autre. Le résultat est que les situations sont présentées différemment et que cela semble prétériter, du point de vue sémantique, l'un des deux textes. L'effet du mouvement des marqueurs de réserve au niveau du texte est réalisé par l'insertion, à l'intérieur de l'énoncé général ou au niveau syntagmatique, d'un signe d'hésitation, d'une complétion succincte ou au contraire d'une omission d'information pouvant conduire à un changement de registre sémantique.

Les changements produits au niveau du texte, dus bien souvent à la subjectivité manifestée au moment de la traduction, à la manière dont l'auteur perçoit la mentalité et la culture du peuple dans la langue duquel il écrit, ne sont pas seulement de nature structurelle, mais ils impliquent aussi un changement de perspective qui conduit finalement à une modification non négligeable de l'énoncé dans la langue de traduction. Par conséquent, il s'agit de découvrir les constantes qui permettent

2 Voir Liliana Agache, Traducere sau rescriere? Opera lui Panait Istrati sau arta modulației dans SCL, LI, 1, janvier-juin, 2000, p. 27-32.

3 Pour ce terme, voir Teodora Cristea (2000, 173). 
d'identifier les éléments aidant à l'adaptation. La même unité doit se retrouver dans une grande diversité de manifestations linguistiques.

Bien que l'essence informationnelle contenue dans l'énoncé de la langue originale d'écriture, dans ce cas le français, n'est pas fondamentalement affectée, l'adaptation influera tant sur la structure grammaticale que sur la structure sémantique du texte rédigé en roumain. Les transpositions simples du texte d'une langue dans une autre sont bien sûr les plus fréquentes, mais on repère aussi, à de nombreuses reprises, des extensions sémantiques, des réductions, des groupes incidents, des transpositions ponctuelles et complexes, corrélatives, inversées ou croisées. Nous montrerons ci-dessous quelques exemples de traduction qui vont parfois même jusqu'à trahir une véritable réécriture du texte, par un changement qui implique le déplacement du point de vue de l'incidence sémantique.

Les premiers exemples sont tirés du deuxième chapitre. Dans le texte français, une précision supplémentaire est donnée pour marquer le cadre émotionnel dans lequel l'évènement a lieu - « à l'époque la plus consciente de mon enfance »- cadre qui, dans le texte roumain, est implicite :

\begin{tabular}{ll}
\hline Avant de venir loger dans cette & Inainte de a ne muta în curtea \\
cour, nous avons habité pendant & acestuia, locuisem multii ani de-a \\
plusieurs années, à l'époque la & rândul în Comorofca, la două sute \\
plus consciente de mon & de pași de aici.5(p.156) \\
enfance, dans le quartier de & \\
"Comorofca ", à deux cents pas & \\
d'ici.4(p.21) & \\
\hline
\end{tabular}

Le narrateur poursuit, en français, en évoquant les moments et les lieux exacts de ses déménagements successifs (« à Saint-Georges et à Saint-Démètre, au printemps et en automne »), alors que dans le texte roumain, seule l'action de déménager est indiquée, dépourvue de tout cadre spatio-temporel, comme si l'écrivain racontait son histoire à quelqu'un qui la connaissait.

\begin{tabular}{rr}
\hline Et encore avons-nous passé & Si încă, în ultimii zece ani, am \\
dans ces dix dernières années dans & trecut numai prin două mahalale; \\
deux quartiers seulement ; mais & dar, când eram mic, se întâmpla să \\
quand j’étais petit, il nous arrivait & \\
\hline
\end{tabular}

\footnotetext{
4 Le texte de référence en français est le suivant : Panaït Istrati, La Jeunesse d'Adrien Zograffi. Vol. II : Codine, Mikhaïl, Mes départs, Le pêcheur d'éponges. Paris, Gallimard, 1968.

5 Pour la version roumaine, nous nous référons à l'édition qui reproduit le texte des maisons d'édition Minerva de Bucarest de 1987 : Panait Istrati, Chira Chiralina. Codin. Ciulinii Bărăganului. București, Curtea Veche, 2009.
} 
de déménager deux, trois fois par

an : à Saint-Georges et à Saint-

Démètre, au printemps et en

automne. (p.21) ne mutăm de două, de trei ori pe an. (p. 156)

Pour décrire la façon dont la mère d'Adrien s'y prend pour trouver sans cesse de nouveaux pieds-à-terre, le texte français renchérit sur son acharnement à l'aide d'une comparaison destinée à en donner une image plus explicite : " comme une lionne ». Cette comparaison disparaît dans le texte roumain.

\begin{tabular}{ll}
\hline Quelques semaines avant le & \multicolumn{1}{c}{ Cu câteva săptămâni înainte de } \\
terme, elle s'en allait comme une termen, cutreiera împrejurimile, cu \\
lionne battre la banlieue, le nez en nasul în vânt, pentru a zări \\
l'air pour apercevoir l'énigmatique & enigmaticul afiș. Cuvintele «de \\
petite affiche dont les deux mots «À & inchiriat»le deslușea ca și cum ar fi \\
Louer »lui étaient aussi clairs qu'ils & știut să citească. (p. 156) \\
l'auraient été à une personne & \\
sachant lire. $(p .21-22)$ &
\end{tabular}

Le passage qui suit décrit une méthode locale (roumaine) de nettoyage et d'assainissement des logements. On ne sera donc pas étonné que la description soit relativement détaillée en français, car il faut expliquer au lecteur non familier de ces pratiques de quoi il s'agit, ce qui n'est pas le cas dans la version roumaine. Ainsi, là où le français a besoin de toute une phrase telle que " enduisait le sol avec de la glaise jaune mêlée de boue », le roumain n'a besoin que de trois mots lipea pe jos, ce qui signifie littéralement " coller au sol », le verbe a lipi ( coller ») suffisant à lui seul pour tout dire. Notons en outre que la charge sémantique de ce verbe roumain insiste aussi l'aspect émotionnel de l'action.

\begin{tabular}{|c|c|}
\hline $\begin{array}{l}\text { Elle lavait la boiserie à l'eau } \\
\text { chaude, pourchassait les punaises, } \\
\text { arrachait les clous innombrables, } \\
\text { bouchait - avec du savon, du soufre } \\
\text { et de la pâte empoisonnée-toutes les } \\
\text { fentes des murs et les trous de souris, } \\
\text { passait deux couches de chaux } \\
\text { blanche sur le mur et enduisait le } \\
\text { sol avec de la glaise jaune } \\
\text { mêlée de boue... (p. 22) }\end{array}$ & $\begin{array}{l}\text { Opărea cu apă clocotită tot ce } \\
\text { era lemnărie, ca să stârpească } \\
\text { ploșnițele, scotea nenumărate cuie } \\
\text { infipte în pereți, astupa cu săpun și } \\
\text { pucioasă crăpăturile zidurilor și } \\
\text { găurile șoarecilor, văruia pereții de } \\
\text { două ori și lipea pe jos. ( } p .156-157)\end{array}$ \\
\hline
\end{tabular}

L'adaptation est la solution privilégiée par le roumain lorsqu'il s'agit de faire fi de certaines complétions périphrastiques demandées ou extrapolées par le français, sans rien enlever par ailleurs au sens du fragment. Dans ce passage, le proverbe français destiné à encadrer 
l'action, « à tout seigneur tout honneur », est passé sous silence dans la version roumaine, de même que la précision de l'instrument avec lequel le crâne a été fendu ( « avec une bouteille »).

\begin{tabular}{ll}
\hline Ils se rappelaient, soudain, & \multicolumn{1}{c}{ Iși aduceau aminte, așa } \\
qu'une vraie partie de plaisir n'a & deodată, că o petrecere nu are \\
point de piquant sans quelques & haz, fără fălci rupte, capete \\
mâchoires de travers, un crâne & sparte, câte un ochi scos sau câte \\
fendu avec une bouteille, un oeil & un nas pocit. $(p .164)$ \\
ou un nez rendus méconnaissables; & \\
- enfin (à tout seigneur tout & \\
honneur!) etc. (p. 33$)$ & \\
\hline
\end{tabular}

En d'autres occasions, on peut observer que l'auteur fait des choix complètement différents, préférant une variante métaphorique à une description technique ou factuelle. C'est ainsi que le quartier de Comorofca qui, dans la version française, est simplement qualifié de « mal famé ", se teinte d'une charge émotionnelle beaucoup plus puissante en roumain, où il devient un quartier « célèbre pour ses couteaux » (vestită prin cuțitarii ei).

\begin{tabular}{ll}
\hline - Voilà, mon enfant! C'est fait... & - Așa, băiatu' mamiï! Am \\
Nous sommes descendus un rang & făcut-oșipe asta... Ne-am coborât \\
plus bas... Nous voilà dans la & o treaptă mai jos... Iată-ne și în \\
Comorfca, le quartier le plus & Comorofca, cea vestită prin \\
malfamé de la banlieue. $(p .22)$ & cuțitarii ei. $(p .157)$ \\
\hline
\end{tabular}

Lorsque, dans la version française, Codine raconte son histoire à Adrien, il la ponctue d'expressions pour s'assurer de l'attention de l'enfant : descriptions ekphrastiques propres à ébranler son imagination (« il était fort à éclater par trop de sang »), ou pauses phatiques et adresses explicites qui l'intègrent dans son récit (« Eh ! mon enfant ! »). Rien de tel dans la version roumaine, beaucoup plus concise, mais où le rythme des phrases et les mots utilisés n'en produisent pas moins le même effet. La version française, de manière générale, montre des locuteurs préoccupés de la réaction de leurs interlocuteurs, certes pour les solliciter, mais aussi pour les ménager : l'enfant se tait « sur tout ce qui pouvait l'effrayer [sa mère] ».

\begin{tabular}{|c|c|}
\hline $\begin{array}{l}\text { Il avait dix-huit ans, il était } \\
\text { fort à éclater par trop de sang } \\
\text { et travaillait dans une équipe de } \\
\text { terrassiers pour la construction de } \\
\text { la route - Eh ! mon enfant !... } \\
\text { Que de choses tristes !... Tous le } \\
\text { haïssaient !... A cause de sa } \\
\text { force !... (p. 47) }\end{array}$ & $\begin{array}{l}\text { Avea optsprezece ani, era } \\
\text { voinic ca un taur și muncea într-o } \\
\text { echipă de lucrători la facerea } \\
\text { șoselei. - Eh, câte lucruri triste!... } \\
\text { Toți îl urau, din pricina tăriei } \\
\text { lui!... (p. 175) }\end{array}$ \\
\hline
\end{tabular}


Il ne m'en fallut pas plus pour perdre la tête ; et, le soir me taisant sur tout ce qui pouvait l'effrayer, j'annonçai à ma mère que le lendemain j’irais chasser avec Codine. (p.58)

Le fragment suivant montre à nouveau un personnage soucieux de l'autre : alors que le narrateur, en français, passe le dimanche " près d'elle » (donc près de sa mère, étant donné qu'il vient d'expliquer que celle-ci éprouvait de la joie à avoir ses heures de repos en sa compagnie), en roumain, il passe la journée dominicale simplement « à la maison ». Néanmoins, une phrase plus loin, lorsque l'amour maternel est évoqué, il n'est pas qualifié dans la version française, alors que dans la version roumaine, il est caractérisé comme étant tiranică - terme dont il est difficile de donner un équivalent en français car même si, littéralement, il signifie „tyrannique”, les connotations du terme dans les deux langues ne coïncident pas. Dans ce contexte, le mot se manifeste en roumain comme le résultat d'une perception qui se produit au croisement de la compétence linguistique et de l'aspect culturel, et il désigne un superlatif absolu positif fréquemment utilisé en roumain.

Je passais donc mes dimanches près d'elle ; il n'y avait que le jeudi pour m'emplir la poitrine de ce souffle divin qui est la conscience d'être entièrement libre, même de l'amour de sa mère. ( $p$. 35-36)
Stând deci, toate duminicile acasă, nu-mi rămânea decât joia ca să mă îmbăt cu acel har dumnezeiesc care este conștiința de-a fi cu totul liber, descătușat chiar de dragostea tiranică $a$ mamei. ( $p .167)$

La subjectivité affective se manifeste également par l'ajout de certains marqueurs dans le texte en roumain, qui n'ont pas été jugés nécessaires dans la première version en français. On remarque en effet en roumain des fins de phrases qui n'apparaissent pas dans le texte français, comme dans ces deux passages : l'ange est envoyé " pour lui tenir compagnie » (ca să-i ție tovărășie) tandis qu'il traverse le Danube « dans une canicule étouffante » (pe un zăduf mare) :

Pendant dix ans il a tiré du sel dans les mines. Les gens les surnomment «le forçat ». Ils ont tort. La preuve que Dieu veut être maintenant bien gracieux avec lui, c'est qu'il a envoyé un ange innocent comme toi... (p. 47)
Zece ani a tăiat sare în ocnă. oamenii îi spun " ocnașul ». N-au dreptate. Dovadă e că Dumnezeu e bun cu el și-i trimite acum un înger nevinovat, ca tine, ca să-i tie tovărășie. (p. 175) 
...puis on se dit adieu. Et à une

heure de l'après-midi, nous

repassions le Danube. ( $p .47)$
Apoi ne-am despărțit. La unu după-amiază treceam Dunărea, pe un zăduf mare. (p. 176)

Les options différentes adoptées lors de la narration descriptive sont frappantes, et elles sont souvent fortement tributaires de la culture et des moeurs dans lesquelles s'inscrivent les deux langues, comme c'est le cas dans l'exemple qui suit : alors que dans la version française, il est dit que tous sont « bien habillés » et qu'il y a « des fleurs partout », la version roumaine recourt à une expression toute faite en disant que tous sont « bien habillés », et que " certains tenaient un géranium entre leurs dents » - la dernière expression relevant de la culture populaire roumaine.

\begin{tabular}{ll}
\hline Tous, bien habillés, mais leurs & \multicolumn{1}{c}{ Toți erau bine îmbrăcați, deși } \\
vêtements maculés. Les chapeaux & aveau hainele pătate. Purtau \\
sur la nuque ou sur le front, sur & pălăriile pe ceafă, pe frunte sau pe- \\
une oreille ou sur l'autre, et des & $\begin{array}{l}\text { o ureche, iar unii țineau între } \\
\text { dințio mușcată. }(p .177)\end{array}$ \\
fleurs partout. $(p .49)$ &
\end{tabular}

D'autres éléments de l'adaptation sont marqués par l'emploi dans le texte roumain de certains syntagmes qui semblent totalement indépendants du contexte - telle que la phrase « mais on ne doit pas se fâcher pour si peu de chose » (Dar nu trebuie să ne supărăm pentru atâta lucru), qui rééquilibrent la structure du contexte au niveau affectif.

- Tiens ! les eaux sont plus basses que je ne l'avais prévu !... Si le ciel se maintient serein, nous aurons bientôt clair de lune... ce sera plus facile et plus agréable. (p. 59)
- Ei, drăcie, apele sunt mai mici decât credeam!... Dar nu trebuie să ne supărăm pentru atâta lucru. Dacă cerul rămâne senin, va ieși în curând luna plină... Atunci va fi mai plăcut și mai ușor de înaintat. (p.184)

La scène du dernier crime de Codin est racontée différemment dans les deux versions, à partir du moment où la veilleuse est allumée par la tenancière du cabaret : la version roumaine contient des adverbes de manière et de lieu - « elle court rapidement à côté », d'où elle revient avec une " chandelle à laquelle elle ajoute du pétrole ». Après avoir changé la mèche, deux actions ont lieu dans la version roumaine - elle " allume » [la chandelle] (aprinde) et « se met à prier » (se pune pe închinat).

- On n'a pas allumé la veilleuse pour la miséricordieuse Sainte Vineri !...

Elle court, revient avec un verre et se met à préparer la
- N-am aprins candela pentru milostiva Sfânta Vineri!

Dă fugulița alături și se întoarce cu candela, îi mai adaugă untdelemn, schimbă 
veilleuse, ajoutant de l'huile et changeant la mèche. (p. 81) iasca, aprinde și se pune pe inchinat. (p. 201)

De même, le texte roumain est plus précis dans la description, par le narrateur, du regard de la mère de Codine une heure avant son crime, et il donne ainsi des indices supplémentaires annonçant le meurtre de son fils : ce regard, avant de fixer avec mépris le narrateur, s'attarde tout d'abord sur la bouche de Codine, information absente du texte français. Or, la vieille donnera la mort à son fils endormi en déversant deux litres d'huile bouillante justement dans sa bouche.

\begin{tabular}{ll}
\hline Alors je vis la mère Anastasie & Atunci, o văzui pe mama \\
lever la tête avec dureté. Ses & Anastasia ridicând capul cu \\
regards, furtifs d'ordinaire, & asprime. Privirea ei, furișă de \\
fixèrent mon visage avec mépris. & obicei, se opri mai întầi asupra \\
$(p .84)$ & $\begin{array}{l}\text { gurii lui Codin, apoi asupra } \\
\text { mea, plină de dispreț. }(p .204)\end{array}$ \\
\hline
\end{tabular}

D'autres détails descriptifs font également défaut dans le texte français, tels « le cœur écrasé » et « le sang pulsant violemment dans la tête » du narrateur, qui trahissent son âme déchirée quand il entre pour la dernière fois dans la cour de la maison de Codine et le voit mort, avec un sac couvrant son corps - ou tel encore le regard de la mère de Codine, qui contemple le cadavre de son fils « comme on contemple un vers ».

Les jambes défaillantes, me traînant, j'entrai chez Codine par la porte de la rue. (p. 85)
Impleticindu-mă, ca un om beat, cu inima încleștată, cu capul vâjâind, intrai la Codin pe poarta de la stradă. (p. 205)
À côté, sur la " prispa », la mère Anastasie était accroupie près $d u$ cadavre de son fils, un cierge de cinq centimes à la main. Elle le contemplait. La flamme du cierge vacillait au vent. (p. 86)
Alături, pe prispă, mama Anastasia se ghemuise lângă cadavrul fiului ei, cu o lumânare de cinci parale în mână. $\hat{\boldsymbol{I}}$ privea cum privești o râmă. Flacăra lumânării pâlpâia în adierea vântului rece. (p. 205)

La différence la plus importante entre les deux versions est sans doute celle que l'on trouve dans un passage se situant à peu près au milieu du roman, et qui narre la façon dont se déroule le recrutement des ouvriers : là où le texte en français décrit les actions de Codine en quelques phrases ( « Je voyais Codine (...) imposer leur encadrement dans les équipes en formation. Il le faisait sèchement, avec une mine si féroce que je sentais la douleur me tenailler le cœur »), le texte roumain rallonge sensiblement la description et rajoute même des prises de parole. Au 
total, tout ce fragment est inséré : Codine « distribuait un sac à chacun d'eux, et ils l'attrapaient comme des petits pains frais. D'autres criaient : "Et moi, Codine et moi, parce que mes enfants meurent de faim !" Codine, la mâchoire serrée, fronça les sourcils, l'examina un instant et lança son sac, tandis que l'homme se dandinait et se tut ». La version roumaine met beaucoup mieux en évidence que la française toute l'humanité qui se cache sous les manières dures et cassantes de l'ancien forçat.

\begin{tabular}{|c|c|}
\hline $\begin{array}{l}\text { Avec une stupéfaction } \\
\text { grandissante, je voyais Codine } \\
\text { appeler plusieurs manouvres } \\
\text { miséreux, et imposer leur } \\
\text { encadrement dans les équipes en } \\
\text { formation. Il le faisait sèchement, } \\
\text { avec une mine si féroce que je } \\
\text { sentais la douleur me tenailler le } \\
\text { cour. } \\
\text { Puis, il sortit du groupe et } \\
\text { revient avec moi en un endroit } \\
\text { isolé. }(p .42)\end{array}$ & $\begin{array}{l}\text { Din ce în ce mai uimit, îl văzui } \\
\text { pe Codin chemând mai mulți } \\
\text { hamali amărâți și distribuind } \\
\text { fiecăruia câte un sac, pe care } \\
\text { ei îl apucau ca pe o pâine } \\
\text { caldă. Alții strigau: } \\
\text { - Și mie, Codin, și mie, căa-mi } \\
\text { mor copiii de foame! } \\
\text { Codin, cu fălcile încleștate, } \\
\text { se uita încruntat în jos, îl } \\
\text { examina o clipă și-i arunca } \\
\text { sacul, în timp ce vắtaful turba } \\
\text { și tăcea chitic. Când termină, } \\
\text { Codin ieși din mulțime și mă luă cu } \\
\text { el într-un loc singuratec. ( } p .172 \text {. }\end{array}$ \\
\hline
\end{tabular}

\section{Conclusion}

En comparant les deux versions de Codine de Panaït Istrati, on constate que la subjectivité du narrateur se manifeste linguistiquement de manière différente dans les deux textes.

Dans le texte original français, l'auteur est parfois plus prolixe que dans le texte rédigé dans l'après-coup dans sa langue maternelle, en particulier quand il s'agit d'évoquer des habitudes ou traditions typiques de la société roumaine, mais aussi peut-être par contamination de pratiques littéraires françaises témoignant d'un souci plus poussé d'analyse ou d'introspection. Les précisions, s'imposant par des explications supplémentaires, des comparaisons, des incises entre parenthèses, parfois même par des modifications du discours, trahissent la préoccupation de l'auteur d'être bien compris par les locuteurs de sa langue d'adoption. Parfois au contraire, c'est la version roumaine qui est plus éloquente, surtout quand il s'agit de rendre compte d'une atmosphère locale (travail au port), que le lecteur roumain aura sans doute du plaisir à reconnaître, ou encore quand il s'agit d'aiguiser à l'extrême certains constrastes (dans la personnalité de Codine par exemple), ou encore quand il s'agit de suggérer une scène ou une situation particulièrement intolérable (la haine de la mère pour son fils) - scènes ou situations qui, par ailleurs, trouvent souvent un 
contrepoint dans la grande tendresse que libère le point de vue du narrateur.

Le fait que Panaït Istrati semble réécrire certains fragments de son roman plutôt que de les traduire constitue la preuve de sa difficulté à reproduire littéralement une réalité linguistique familière dans une autre langue familière, ainsi que les réalités culturelles qu'elles manifestent. L'adaptation a lieu par l'expression d'une subjectivité en léger décalage dans les deux textes. Mais il apparaît avec évidence que Panaït Istrati éprouve un plaisir quasi sensuel à transposer son texte du français dans sa langue maternelle, car la version en roumain vibre d'une charge émotionnelle (positive ou négative) beaucoup plus puissante.

\section{Bibliographie}

Bouton, Charles (1978). La linguistique appliquée. Paris : PUF.

Cristea, Teodora. (1977). Éléments de grammaire contrastive. Bucureşti: Editura Didactică şi Pedagogică.

Cristea, Teodora. (1982). Contrastivité et traduction. Bucureşti: Tipografia Universității Bucureşti.

Cristea, Teodora. (2000) Stratégies de la traduction. Bucureşti : Ed. Fundației România de Mâine.

Delavenay, Emile. (1959). La machine à traduire. Paris : PUF.

Jutrin-Klener, Monique. (1970). Panaït Istrati, Un chardon déraciné. Paris : Maspero.

Lerault, Daniel. (1987). " Panaït Istrati. À propos d'une récente acquisition de la Bibliothèque Nationale », Revue de la Bibliothèque Nationale, 24 : 14-25.

Mounin, Georges. (1976). Linguistique et traduction. Bruxelles : Dessart et Mardaga.

\section{Textes de référence}

Panait Istrati. (2009). Chira Chiralina. Codin. Ciulinii Bărăganului. București : Curtea Veche.

Panaït Istrati. (1968). Codine. La Jeunesse d'Adrien Zograffi. Vol. II : Codine, Mikhaïl, Mes départs, Le pêcheur d'éponges. Paris : Gallimard. 